\title{
Identification and Disease Index Inversion of Wheat Stripe Rust and Wheat Leaf Rust Based on Hyperspectral Data at Canopy Level
}

\author{
Hui Wang, ${ }^{1}$ Feng Qin, ${ }^{1}$ Qi Liu, ${ }^{1}$ Liu Ruan, ${ }^{1}$ Rui Wang, ${ }^{2}$ Zhanhong Ma, \\ Xiaolong Li, ${ }^{1}$ Pei Cheng, ${ }^{1}$ and Haiguang Wang ${ }^{1}$ \\ ${ }^{1}$ College of Agriculture and Biotechnology, China Agricultural University, Beijing 100193, China \\ ${ }^{2}$ Kaifeng Experimental Station of China Agricultural University, Kaifeng 475004, China
}

Correspondence should be addressed to Haiguang Wang; wanghaiguang@cau.edu.cn

Received 9 March 2015; Accepted 8 June 2015

Academic Editor: Gianfranco Giubileo

Copyright ( $) 2015$ Hui Wang et al. This is an open access article distributed under the Creative Commons Attribution License, which permits unrestricted use, distribution, and reproduction in any medium, provided the original work is properly cited.

\begin{abstract}
Stripe rust and leaf rust with similar symptoms are two important wheat diseases. In this study, to investigate a method to identify and assess the two diseases, the canopy hyperspectral data of healthy wheat, wheat in incubation period, and wheat in diseased period of the diseases were collected, respectively. After data preprocessing, three support vector machine (SVM) models for disease identification and six support vector regression (SVR) models for disease index (DI) inversion were built. The results showed that the SVM model based on wavelet packet decomposition coefficients with the overall identification accuracy of the training set equal to $99.67 \%$ and that of the testing set equal to $82.00 \%$ was better than the other two models. To improve the identification accuracy, it was suggested that a combination model could be constructed with one SVM model and two models built using $K$ nearest neighbors $(K \mathrm{NN})$ method. Using the DI inversion SVR models, the satisfactory results were obtained for the two diseases. The results demonstrated that identification and DI inversion of stripe rust and leaf rust can be implemented based on hyperspectral data at the canopy level.
\end{abstract}

\section{Introduction}

Stripe rust caused by Puccinia striiformis f. sp. tritici (Pst) and leaf rust caused by $P$. recondita $\mathrm{f}$. sp. tritici (Prt) are two devastating wheat diseases worldwide [1]. They occur widely in wheat growing regions in China and they are the major obstacles to stable and high yield wheat production [1-3]. In China, a couple of severe epidemics of the two diseases occurred and destructive yield losses of wheat were caused [1-3]. These two diseases are easily confused with each other because of the similar disease symptoms. So it is difficult to make an accurate diagnosis and monitoring of the diseases under field conditions. Thus timely management of the diseases may be affected. Traditionally, the identification and assessment of the two diseases mainly rely on the naked-eye field observation and investigation of the visible disease symptoms conducted by plant protection technical personnel. But this method is labor-consuming and time-costing, and it easily results in errors. Therefore, it is of great significance to explore a method for rapid and accurate identification and quantitative assessment of these two diseases.

The rapid development of remote sensing technology provides strong technical support for the monitoring of plant diseases. The real-time, objective, rapid, and nondestructive methods for identifying, detecting, and monitoring plant diseases may be developed based on remote sensing technology. Remote sensing techniques have been applied in the studies on identifying and monitoring plant diseases [46]. Remote sensing technology, especially the hyperspectral remote sensing technology, has been applied to remote sensing monitoring of wheat stripe rust [7]. Detection and identification of wheat stripe rust via remote sensing at the individual wheat leaves [8-10], monitoring of the disease via ground remote sensing [11-13], aerial remote sensing [14-16], and space remote sensing [17-19] have been investigated. 
Few studies on the detection and disease severity estimation of wheat leaf rust using the spectral reflectance data were carried out at the leaf scale $[20,21]$. At this present time, remote sensing techniques are mostly used in the studies on detecting and monitoring of single plant disease, and there are only few studies on the identification and monitoring of two or more than two kinds of plant diseases. There are few reports focusing on the distinction between wheat stripe rust and other wheat diseases based on remote sensing technology. Devadas et al. [22] evaluated ten widely used spectral vegetation indices for discriminating individual wheat leaves infected with stripe rust, leaf rust, and stem rust caused by the pathogen Puccinia graminis f. sp. tritici (Pgt). The results showed that no single index could discriminate the three kinds of wheat rusts from each other. However, a method for discriminating the three wheat rusts was provided. Firstly, the anthocyanin reflectance index (ARI) could be used to discriminate healthy leaves, leaves infected with stripe rust, and mixed leaves infected with stem rust and leaf rust, and the transformed chlorophyll absorption and reflectance index (TCARI) could then be used to discriminate the leaves infected with leaf rust and those infected with stem rust. In a study conducted by Yuan et al. [23], based on the spectra of individual wheat leaves infected with stripe rust and powdery mildew (caused by Blumeria graminis $\mathrm{f}$. sp. tritici), the model for discriminating the two diseases with the overall accuracy more than $80 \%$ was developed using the selected spectral bands and spectral features, and the retrieving model of disease severity for each disease with the root-mean-square error (RMSE) less than 15\% was also built. The study on identification of damage symptoms caused by powdery mildew, stripe rust, and wheat aphid on winter wheat was conducted by Qiao et al. [24] based on the canopy hyperspectral remote sensing data. The results showed that, after logarithmic-differential transformation of the original spectra, the three kinds of wheat pests were well identified with the accuracies more than $90 \%$ by using stepwise discriminate analysis and hierarchical clustering, respectively. Based on the near-infrared spectral data of individual wheat leaves, $\mathrm{Li}$ et al. [25] investigated the early diagnosis of stripe rust and leaf rust in incubation period and diseased period using near-infrared reflectance spectroscopy (NIRS) technology. The results showed that the identification accuracy of the training set was $97.00 \%$ and the identification accuracy of the testing set was $96.00 \%$ for the optimal model to identify the diseases built by using distinguished partial least squares (DPLS). To the best of our knowledge, at the present time, there are no reports about identifying wheat stripe rust and wheat leaf rust by using hyperspectral remote sensing technology at the wheat canopy level, and there are also no reports about the assessment of wheat leaf rust by using hyperspectral remote sensing technology at the canopy level.

In this study, through the field experiment, identification and disease index inversion of wheat stripe rust and wheat leaf rust were investigated by using hyperspectral remote sensing technology at the canopy level. The aim of this study was to provide a method for monitoring and evaluating the two diseases.

\section{Materials and Methods}

2.1. Field Experiment Design and Artificial Spray Inoculation. Field experiment was conducted in Kaifeng Experimental Station of China Agricultural University, Kaifeng, Henan Province, China. Beijing 0045, a wheat variety which is moderately susceptible to Pst and Prt, was selected as the experimental cultivar. The seeds of the cultivar were sown in the autumn of 2013. The experimental field was divided into two large zones. One zone was designed as the experimental zone of wheat stripe rust, and another was designed as the experimental zone of wheat leaf rust. Each zone was divided into 18 experimental plots. The size of each plot was $3 \mathrm{~m} \times$ $4 \mathrm{~m}$. Wheat variety Nongda 195 was planted as protective belts in the areas between the plots. To get different disease prevalence of wheat stripe rust and wheat leaf rust in the plots, the spore suspensions at five concentrations were prepared for artificial spray inoculation of Pst and Prt, respectively. The concentrations of Pst spore suspensions were $100 \mathrm{mg} / \mathrm{L}$, $80 \mathrm{mg} / \mathrm{L}, 60 \mathrm{mg} / \mathrm{L}, 40 \mathrm{mg} / \mathrm{L}$, and $20 \mathrm{mg} / \mathrm{L}$, respectively, and those of Prt spore suspensions were $50 \mathrm{mg} / \mathrm{L}, 40 \mathrm{mg} / \mathrm{L}$, $30 \mathrm{mg} / \mathrm{L}, 20 \mathrm{mg} / \mathrm{L}$, and $10 \mathrm{mg} / \mathrm{L}$, respectively. The control plots were not inoculated using the pathogens. Each treatment was replicated three times. The experiments were performed with randomized block design. Late in the afternoon in April 2014, the plots that need to be inoculated were evenly sprayed using spore suspensions. For moisturizing the wheat leaves, after inoculation, the plots were covered with plastic film that was sprayed with water, and then the edges of the plastic film were covered with earth. Between 8:00 and 9:00 (Beijing Time) in the next day, the plastic film was unveiled.

2.2. Acquisition of Hyperspectral Data. The canopy hyperspectral data of wheat were measured by using an ASD spectrometer (ASD FieldSpec HandHeld 2) (ASD Inc., Boulder, CO, USA) with a wavelength range of 325-1075 nm, a wavelength accuracy of $\pm 1 \mathrm{~nm}$, a spectral resolution of $<3 \mathrm{~nm}$ at $700 \mathrm{~nm}, 25^{\circ}$ field-of-view, and minimum integration time of $8.5 \mathrm{~ms}$. The hyperspectral data were acquired before inoculation (wheat was healthy) and in incubation period of the diseases (wheat has been infected with the pathogen spores, but no disease symptoms were visible) and also were acquired in diseased period (in this period, the data acquisition was conducted twice on April 14, 2014, and on April 29, 2014, resp.). All measurements of hyperspectral data were carried out on clear, sunny days between 10:00 and 14:00 (Beijing Time). In each experimental plot, 5 points (fivepoint sampling) were marked and treated as fixed points for hyperspectral measurements. The spectrum average was set as 15. Three spectra were measured for the wheat canopy at each marked point, and the average value was treated as the canopy spectrum at the point. When the hyperspectral measurement was taken, the sensor of the spectrometer was vertically positioned at a height of $1.3 \mathrm{~m}$ above ground. White board correction was performed before hyperspectral measurements in each plot. A total of 602 reflectance spectra were used for subsequent analysis. The spectra included 210 canopy spectra of healthy wheat (90 spectra obtained in the experimental zone of wheat stripe rust before inoculation, 
90 spectra obtained in the experimental zone of wheat leaf rust before inoculation, 15 spectra obtained in the control plot of wheat stripe rust in incubation period, and 15 spectra obtained in the control plot of wheat leaf rust in incubation period), 75 canopy spectra of wheat in incubation period of stripe rust, 75 canopy spectra of wheat in incubation period of leaf rust, 92 canopy spectra of wheat in diseased period of stripe rust, and 150 canopy spectra of wheat in diseased period of leaf rust. In the diseased period of stripe rust, 150 canopy spectra of the inoculated wheat were obtained. To avoid the effects of the spectra with the disease indices equal to 0 on disease identification and assessment, 92 spectra with the disease indices greater than 0 were used for the corresponding analysis. In the diseased period of leaf rust, 150 canopy spectra of the inoculated wheat were also obtained. The corresponding disease indices were all greater than 0 , so all of the spectra of the diseased wheat infected with leaf rust were used for the corresponding analysis.

At the same time as the spectra were acquired, wheat stripe rust and leaf rust were assessed according to the Rules for Monitoring and Forecast of the Wheat Stripe Rust (Puccinia striiformis West.) (National Standard of the People's Republic of China, GB/T 15795-2011) and the Rules for the Investigation and Forecast of Wheat Leaf Rust (Puccinia recondita Rob. et Desm.) (Agricultural Industry Standard of the People's Republic of China, NY/T 617-2002), respectively. For wheat stripe rust and leaf rust, disease severity of the diseased leaf was estimated as $1 \%, 5 \%, 10 \%, 20 \%, 40 \%, 60 \%$, $80 \%$, or $100 \%$. Disease index (DI) at each marked point was calculated by using the following formula:

$$
\mathrm{DI}=I \times \bar{S} \times 100,
$$

where $I$ was disease incidence and $\bar{S}$ was average disease severity. $\bar{S}$ was calculated by using the following formula:

$$
\bar{S}=\frac{\sum\left(S \times n_{i}\right)}{n} \times 100,
$$

where $\bar{S}$ was average disease severity, $S$ was disease severity, $n$ was the total number of diseased leaves, and $n_{1}, n_{2}, n_{3}, \ldots, n_{8}$ were the number of diseased leaves with disease severity as $1 \%, 5 \%, 10 \%, \ldots, 100 \%$, respectively.

2.3. Preprocessing Methods of Hyperspectral Data. To mine information from the acquired hyperspectral data, the first derivatives and the second derivatives of the original spectral reflectance data were calculated by using the Savitzky-Golay method [32] with the polynomial degree equal to 3 and the span equal to 7. And then the original spectral reflectance data, the Savitzky-Golay first derivatives, and the SavitzkyGolay second derivatives were regarded as the first group of spectral features. Wavelet packet analysis (WPA) was used as a method for spectral data preprocessing in this study. As a time-frequency analysis method, WPA is better than wavelet analysis method, and using the former, high frequency signals and low frequency signals can be simultaneously decomposed [33]. So full-channel signal decomposition can be implemented using WPA. In this study, two-level wavelet packet decomposition of the original spectral reflectance data, the Savitzky-Golay first derivatives, and the SavitzkyGolay second derivatives were simultaneously performed by using db5 wavelet. And then a total of 2328 coefficients of the second level wavelet packet decomposition were chosen as the second group of spectral features. Based on the original canopy spectral data of wheat, a total of 25 feature parameters (as shown in Table 1) were obtained and then were regarded as the third group of spectral features. Considering there were great differences between the magnitudes of the different feature parameters in the third group, the feature parameters were normalized to $0-1$ by using the formula $x_{i}=\left(X_{i}-\right.$ $\left.X_{\min }\right) /\left(X_{\max }-X_{\min }\right)$, where $x_{i}$ was the parameter value after normalization, $X_{i}$ was the parameter value before normalization, $X_{\min }$ was the minimum value of the parameter, and $X_{\max }$ was the maximum value of the parameter. And the normalized values were then used for modeling.

\subsection{Identification of Wheat Stripe Rust and Leaf Rust Based} on Canopy Hyperspectral Data. The canopy spectral data of healthy wheat, wheat in incubation period of stripe rust, wheat in diseased period of stripe rust, wheat in incubation period of leaf rust, and wheat in diseased period of leaf rust were treated as one category, respectively. Thus the spectral data were divided into five categories. The KennardStone method [34] was used to choose samples to constitute training set and testing set for modeling. With the point nearest to the center point as the starting point and the Euclidean distance as the dissimilarity measure, 30 samples were selected from each category to set up testing set, and the rest were treated as training set. In particular, for the third group of spectral features, the dimension of the training set was reduced to four using linear discriminant analysis (LDA), and, based on the obtained means and score matrix of the training set, the dimension of the testing set was also reduced to four. Then the establishment of disease identification model was conducted.

In this study, disease identification modeling was conducted using support vector machine (SVM) based on the three groups of spectral features, respectively. SVM can satisfactorily solve the small sample, nonlinear problems, high dimension, local minimum points, and other practical issues, and it has high generalization ability [35]. SVM models for disease identification were established with radial basis function (RBF) as the kernel function by using C-SVM in LIBSVM package developed by Chih-Jen Lin Group from Taiwan, China [36]. For each SVM model, both the optimal penalty parameter $C$ and the optimal kernel function parameter $\gamma$ were searched using grid search algorithm in the range $2^{-10}-2^{10}$ with the searching step equal to 1 . Identification accuracies were calculated at all points within the grid and the values of $C$ and $\gamma$ were selected as the optimal parameters as the identification accuracy of the training set was the highest. Then the SVM models built based on each group of spectral features, respectively, were used to identify the categories of canopy hyperspectral data. The identification accuracy for each category and the overall identification accuracy for all categories were used to evaluate each SVM model. 
TABLE 1: The feature parameters in the third group of spectral features.

\begin{tabular}{|c|c|c|}
\hline Feature parameter & Definition or calculation formula & Reference \\
\hline $\mathrm{Db}$ & The maximum of first derivatives within blue edge $(490-530 \mathrm{~nm})$ & {$[4]$} \\
\hline$\lambda \mathrm{b}$ & Wavelength at the position of $\mathrm{Db}(\mathrm{nm})$ & {$[4]$} \\
\hline Dy & The maximum of first derivatives within yellow edge $(550-582 \mathrm{~nm})$ & {$[4]$} \\
\hline$\lambda y$ & Wavelength at the position of $D y(\mathrm{~nm})$ & {$[4]$} \\
\hline Dr & The maximum of first derivatives within red edge $(680-780 \mathrm{~nm})$ & {$[4]$} \\
\hline$\lambda \mathrm{r}$ & Wavelength at the position of $\operatorname{Dr}(\mathrm{nm})$ & {$[4]$} \\
\hline$R g$ & The maximum reflectance in $510-560 \mathrm{~nm}$ & {$[4]$} \\
\hline$\lambda g$ & Wavelength at the position of $R g(\mathrm{~nm})$ & {$[4]$} \\
\hline$R \mathrm{r}$ & The minimum reflectance in $640-680 \mathrm{~nm}$ & {$[4]$} \\
\hline$\lambda_{0}$ & Wavelength at the position of $R r(\mathrm{~nm})$ & {$[4]$} \\
\hline$S D b$ & Sum of first derivatives within blue edge $(490-530 \mathrm{~nm})$ & {$[4]$} \\
\hline SDy & Sum of first derivatives within yellow edge $(550-582 \mathrm{~nm})$ & {$[4]$} \\
\hline SDr & Sum of first derivatives within red edge $(680-780 \mathrm{~nm})$ & {$[4]$} \\
\hline $\mathrm{Rg} / \mathrm{Rr}$ & The ratio of $R g$ to $R r$ & {$[4]$} \\
\hline$(R g-R r) /(R g+R r)$ & The normalized value of $R g$ and $R r$ & {$[4]$} \\
\hline$S D r / S D b$ & The ratio of $S D r$ to $S D b$ & [4] \\
\hline SDr/SDy & The ratio of $S D r$ to $S D y$ & {$[4]$} \\
\hline$(S D r-S D b) /(S D r+S D b)$ & The normalized value of $S D r$ and $S D b$ & {$[4]$} \\
\hline$(S D r-S D y) /(S D r+S D y)$ & The normalized value of $S D r$ and $S D y$ & {$[4]$} \\
\hline Anthocyanin reflectance index (ARI) & $\mathrm{ARI}=\left(R_{550}\right)^{-1}-\left(R_{700}\right)^{-1}$ & {$[26]$} \\
\hline Chlorophyll absorption ratio index (CARI) & $\begin{array}{l}\text { CARI }=\frac{\left|\left(a \times 670+R_{670}+b\right)\right|}{\sqrt{a^{2}+1}} \times \frac{R_{700}}{R_{670}} \\
\text { in which, } a=\frac{\left(R_{700}-R_{550}\right)}{150}, b=R_{550}-(a \times 550)\end{array}$ & {$[27]$} \\
\hline Photochemical reflectance index (PRI) & $\mathrm{PRI}=\frac{\left(R_{531}-R_{570}\right)}{\left(R_{531}+R_{570}\right)}$ & [28] \\
\hline Plant senescence reflectance index (PSRI) & PSRI $=\frac{\left(R_{678}-R_{500}\right)}{R_{750}}$ & {$[29]$} \\
\hline Triangular vegetation index (TVI) & $\mathrm{TVI}=0.5 \times\left[120 \times\left(R_{750}-R_{550}\right)-200 \times\left(R_{670}-R_{550}\right)\right]$ & {$[30]$} \\
\hline Water index (WI) & $\mathrm{WI}=\frac{R_{900}}{R_{970}}$ & {$[31]$} \\
\hline
\end{tabular}

In this study, if the satisfactory identification accuracies for the SVM models were not obtained, an attempt of $K$ nearest neighbors $(K N N)$ method for disease identification modeling would be conducted. As a nonparametric machine learning algorithm, KNN method has good classification ability and generalization performance [37]. Using $\mathrm{KNN}$ method, the classification is performed based on local information. Compared with SVM, KNN method is more suitable for solving the classification problems with local characteristics.

2.5. DI Inversion of Wheat Stripe Rust and Leaf Rust Based on Canopy Hyperspectral Data. DI inversion models were built by using support vector regression (SVR) for wheat stripe rust and leaf rust, respectively. The spectra with the disease indices greater than 0 acquired in the diseased period of wheat stripe rust or leaf rust were used for building the DI inversion SVR model. The content-grads method [32] was used to choose samples to constitute training set and testing set for modeling.
For the DI inversion SVR model of wheat stripe rust or leaf rust, the ratio of training set to testing set was about $3: 1$. For the SVR model of wheat stripe rust, the training set included 69 spectra and the testing set included 23 spectra. For the SVR model of wheat leaf rust, the training set included 113 spectra and the testing set included 37 spectra.

The DI inversion models of wheat stripe rust were built by using $\varepsilon$-SVR with RBF as the kernel function based on the first group of spectral features, the second group of spectral features, and the third group of spectral features, respectively. In the same way, the DI inversion models of wheat leaf rust were also built. For each SVR model, both the optimal penalty parameter $C$ and the optimal kernel function parameter $\gamma$ were searched using grid search algorithm in the searching range $2^{-8}-2^{8}$ with the searching step equal to 0.8 . Mean squared errors (MSE) were calculated at all points within the grid, and the values of $C$ and $\gamma$ were selected as the optimal parameters as the minimum MSE of the training set was obtained. 


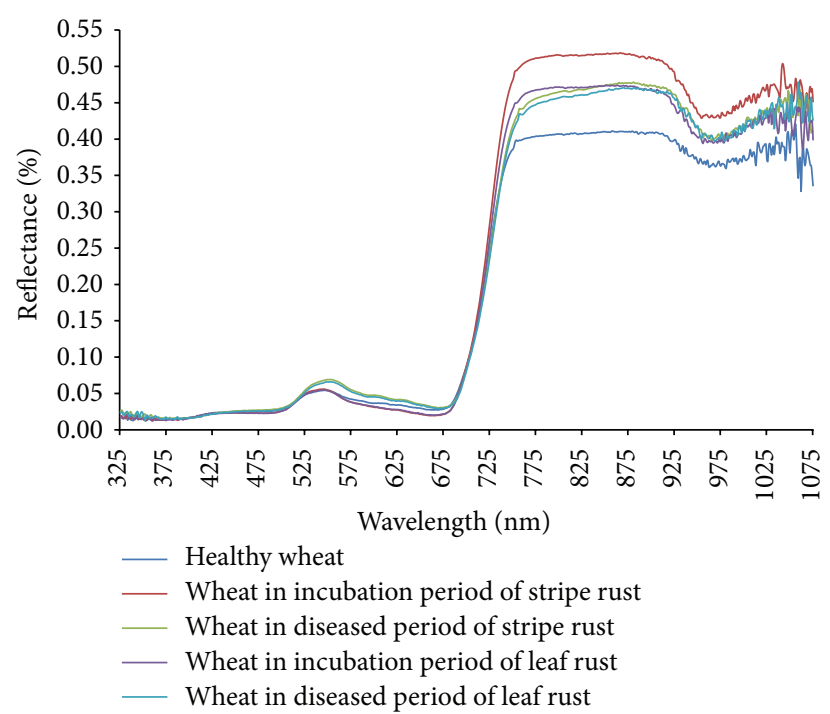

FIgURE 1: The canopy spectra of different categories of wheat.

\section{Results}

3.1. Wheat Canopy Spectra. After averaging the canopy spectral data of healthy wheat, wheat in incubation period of stripe rust, wheat in diseased period of stripe rust, wheat in incubation period of leaf rust, and wheat in diseased period of leaf rust according to their categories, respectively, the spectral curve of each category was shown in Figure 1. As shown in Figure 1, there were great differences between the spectra in the range $720-1075 \mathrm{~nm}$. For wheat stripe rust and leaf rust, the canopy spectral reflectance of wheat in the incubation period and that of wheat in the diseased period relatively increased compared with that of healthy wheat in this spectral range. Both in the incubation period and in the diseased period, the canopy spectral reflectance of wheat infected with Pst relatively increased compared with that of wheat infected with Prt.

3.2. Identification of Wheat Stripe Rust and Leaf Rust. The SVM models for disease identification based on the first group of spectral features, the second group of spectral features, and the third group of spectral features were recorded as Model 1, Model 2, and Model 3, respectively. The optimal parameters $C$ and $\gamma$ for each SVM model and the identification results were shown in Table 2 .

As shown in Table 2, for Model 1, the overall identification accuracies of the training set and the testing set were $97.18 \%$ and $78.00 \%$, respectively. For the testing set, the identification accuracy for wheat in the incubation period of stripe rust was only $20.00 \%$, and the identification accuracies for other categories were more than $80.00 \% ; 23$ spectra of wheat in the incubation period of stripe rust were falsely identified as that of healthy wheat and one spectrum of wheat in the incubation period of stripe rust was identified as that of wheat in the incubation period of leaf rust; two spectra of wheat in the diseased period of stripe rust were identified as that of wheat in the diseased period of leaf rust; one spectrum of wheat in the diseased period of leaf rust was identified as that of wheat in the diseased period of stripe rust.

As shown in Table 2, for Model 2, the overall identification accuracies of the training set and the testing set were $99.67 \%$ and $82.00 \%$, respectively. For the training set, the identification accuracy for healthy wheat was $94.29 \%$ and the identification accuracies for other categories were all $100.00 \%$. For the testing set, the identification accuracy for healthy wheat was $100.00 \%$, that for both wheat in the incubation period of stripe rust and wheat in the diseased period of leaf rust was $86.67 \%$, that for wheat in the diseased period of stripe rust was $63.33 \%$, and that for wheat in the incubation period of leaf rust was $73.33 \%$; two spectra of wheat in the incubation period of stripe rust were falsely identified as that of healthy wheat, one spectrum of wheat in the incubation period of stripe rust was identified as that of wheat in the incubation period of leaf rust, and one spectrum of wheat in the incubation period of stripe rust was identified as that of wheat in the diseased period of leaf rust; 11 spectra of wheat in the diseased period of stripe rust were identified as that of wheat in the diseased period of leaf rust; eight spectra of wheat in the incubation period of leaf rust were identified as that of healthy wheat; four spectra of wheat in the diseased period of leaf rust were identified as that of wheat in the diseased period of stripe rust.

As shown in Table 2, for Model 3, the overall identification accuracy of the training set was $82.89 \%$ and that of the testing set was $68.67 \%$. For the training set, the identification accuracies for healthy wheat, wheat in the incubation period of stripe rust, wheat in the diseased period of stripe rust, wheat in the incubation period of leaf rust, and wheat in the diseased period of leaf rust were $88.33 \%, 82.22 \%, 67.15 \%$, $86.67 \%$, and $88.21 \%$, respectively. For the testing set, the identification accuracy for healthy wheat was $96.67 \%$, that for wheat in the diseased period of leaf rust was $90.00 \%$, that for wheat in the incubation period of leaf rust was $76.67 \%$, and that for wheat in both the incubation period and the diseased period of stripe rust was $40.00 \%$; one spectrum of healthy wheat was identified as that of wheat in the diseased period of leaf rust; 17 spectra of wheat in the incubation period of stripe rust were identified as that of healthy wheat and one spectrum of wheat in the incubation period of stripe rust was identified as that of wheat in the incubation period of leaf rust; 18 spectra of wheat in the diseased period of stripe rust were identified as that of wheat in the diseased period of leaf rust; seven spectra of wheat in the incubation period of leaf rust were identified as that of healthy wheat; three spectra of wheat in the diseased period of leaf rust were identified as that of wheat in the diseased period of stripe rust.

The results showed that Model 1 could effectively distinguish between wheat stripe rust and leaf rust in the diseased period, that Model 2 could effectively separate healthy wheat, wheat in the incubation period of stripe rust, and wheat in the incubation period of leaf rust, and that Model 3 could effectively determine whether wheat was in the diseased period. To improve identification accuracy and modeling effect, it was suggested that the combination model could be constructed. Firstly, the application of Model 3 to determine whether wheat was in the diseased period could 


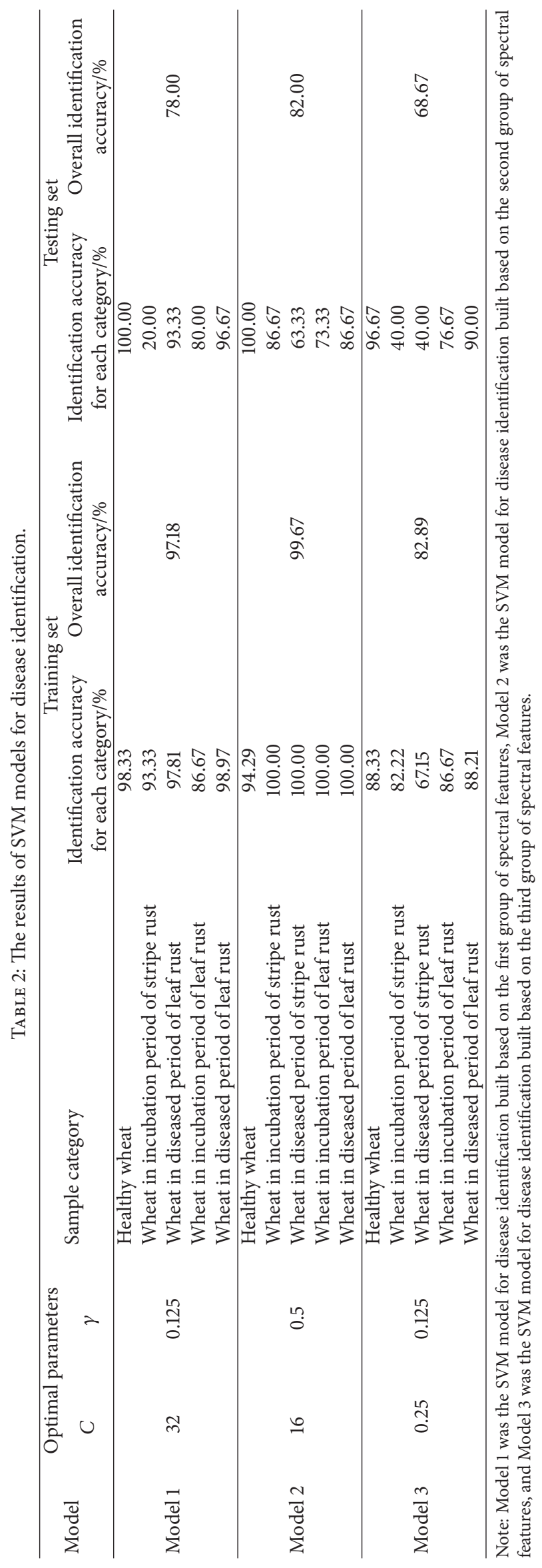


TABLE 3: The results of $K N N$ models for identification of wheat stripe rust and wheat leaf rust.

\begin{tabular}{llccc}
\hline Model & Sample category & $\begin{array}{c}\text { Training set } \\
\text { Identification } \\
\text { accuracy } \\
\text { for each category/\% }\end{array}$ & $\begin{array}{c}\text { Overall } \\
\text { identification } \\
\text { accuracy/\% }\end{array}$ & $\begin{array}{c}\text { Testing set } \\
\text { Identification } \\
\text { accuracy } \\
\text { for each category/\% }\end{array}$ \\
\hline \multirow{2}{*}{ Model 4} & Wheat in diseased period of stripe rust & 100.00 & 100.00 & 100.00 \\
& Wheat in diseased period of leaf rust & 100.00 & 100.00 & 100.00 \\
Model 5 & Healthy wheat & 100.00 & 100.00 & 93.33 \\
& Wheat in incubation period of stripe rust & 100.00 & 83.33 \\
\hline
\end{tabular}

Note. Model 4 was the KNN model built based on the remaining data after deleting the spectra with the disease indices equal to 0 (including the spectra of healthy wheat and the spectra of wheat in the incubation period) from the first group of spectral features, and Model 5 was the KNN model built based on the remaining data after deleting the spectra of wheat in the diseased period from the second group of spectral features.

be performed. If wheat was in the diseased period, Model 1 could be used to distinguish between wheat stripe rust and leaf rust. If not, Model 2 could be used to determine whether wheat was healthy, in the incubation period of stripe rust or in the incubation period of leaf rust.

The results described above indicated that the first group of spectral features reflected the differences between the spectra of wheat in the diseased period of stripe rust and that of wheat in the diseased period of leaf rust, that the second group of spectral features reflected the differences among the spectra of healthy wheat, that of wheat in the incubation period of stripe rust and that of wheat in the incubation period of leaf rust, and that the third group of spectral features reflected the differences between the spectra of healthy wheat and diseased wheat infected with Pst or Prt. For further improving the identification accuracies of models, the spectra with the disease indices equal to 0 (including the spectra of healthy wheat and the spectra of wheat in the incubation period) were deleted from the training set and the testing set used for the establishment of Model 1 , and the rest were treated as the new training set and the new testing set for building the identification model called Model 4 by using $K N N$ method; the spectra of wheat in the diseased period were deleted from the training set and the testing set used for the establishment of Model 2, and the rest were treated as the new training set and the new testing set for building the identification model called Model 5 by using $K \mathrm{NN}$ method. For building $K \mathrm{NN}$ models, the determination of the number of nearest neighbors was performed with Euclidean distance as the dissimilarity measure and the reciprocal of the distance as the weight. Four was determined as the optimal number of the nearest neighbors for Model 4, and five was determined as the optimal number of the nearest neighbors for Model 5. The identification results of Model 4 and Model 5 were shown in Table 3. For Model 4, the overall identification accuracies of the training set and the testing set were both $100.00 \%$. It was indicated that Model 4 could accurately distinguish between wheat stripe rust and leaf rust in the diseased period. For Model 5, the overall identification accuracy of the training set was $100.00 \%$ and that of the testing set was $92.22 \%$. It was demonstrated that Model 5 could separate healthy wheat, wheat in the incubation period of stripe rust, and wheat in the incubation period of leaf rust with high accuracies.

When Model 5 was used to identify the sample categories in the testing set, the spectra of healthy wheat were all identified correctly, two spectra of wheat in the incubation period of stripe rust were identified as that of healthy wheat, and five spectra of wheat in the incubation period of leaf rust were identified as that of healthy wheat. The results showed that, using Model 5, the spectra of healthy wheat and wheat in the incubation period of stripe rust could be confused, the spectra of healthy wheat and wheat in the incubation period of leaf rust also could be confused, and the spectra of wheat in the incubation period of stripe rust and leaf rust could not be confused. However, when Model 2 was used to identify the sample categories in the corresponding testing set, one spectrum of wheat in the incubation period of stripe rust was identified as that of wheat in the incubation period of leaf rust. The results indicated that the $\mathrm{KNN}$ model was more suitable than the SVM model to distinguish the spectra of wheat in the incubation period of wheat stripe rust and leaf rust. Concerning the overall identification accuracies, the identification accuracy for each category, the ability to distinguish the spectra of wheat in the incubation period of stripe rust and leaf rust, and the ability to distinguish the spectra of wheat in the diseased period of stripe rust and leaf rust, it was suggested that Model 1 could be replaced by Model 4, and Model 2 could be replaced by Model 5, and then the combination model could be constructed with Model 3, Model 4, and Model 5 for identification of wheat stripe rust and leaf rust based on canopy hyperspectral data.

3.3. DI Inversion of Wheat Stripe Rust and Leaf Rust. The DI inversion SVR models of wheat stripe rust and leaf rust built based on the spectra with the disease indices greater than 0 acquired in the diseased period in the first group of spectral features were recorded as Model 6 and Model 7, respectively. The DI inversion SVR models of wheat stripe rust and leaf rust built based on the spectra with the disease indices greater than 0 acquired in the diseased period in the second group of spectral features were recorded as Model 8 and Model 9, respectively. The DI inversion SVR models of wheat stripe 
TABLE 4: The results of disease index inversion SVR models of wheat stripe rust and wheat leaf rust.

\begin{tabular}{lcccccrr}
\hline \multirow{2}{*}{ Model } & \multirow{2}{*}{ Wheat disease } & \multicolumn{2}{c}{ Optimal parameters } & \multicolumn{2}{c}{ Training set } & \multicolumn{2}{c}{ Testing set } \\
& & $C$ & $\gamma$ & $R^{2}$ & MSE & 0.0068 & 0.6750 \\
\hline Model 6 & Wheat stripe rust & 32 & 0.03125 & 0.8895 & 0.0193 \\
Model 7 & Wheat leaf rust & 4 & 0.03125 & 0.8435 & 0.0104 & 0.8099 \\
Model 8 & Wheat stripe rust & 32 & 0.03125 & 0.8942 & 0.0062 & 0.6597 & 0.0130 \\
Model 9 & Wheat leaf rust & 4 & 0.03125 & 0.8479 & 0.0101 & 0.8068 \\
Model 10 & Wheat stripe rust & 16 & 0.2500 & 0.8623 & 0.0085 & 0.6250 \\
Model 11 & Wheat leaf rust & 4 & 0.1250 & 0.8461 & 0.0095 & 0.7982 \\
\hline
\end{tabular}

Note. Model 6 was the DI inversion SVR model of wheat stripe rust built based on the spectra with the disease indices greater than 0 acquired in the diseased period of stripe rust in the first group of spectral features. Model 7 was the DI inversion SVR model of wheat leaf rust built based on the spectra with the disease indices greater than 0 acquired in the diseased period of leaf rust in the first group of spectral features. Model 8 was the DI inversion SVR model of wheat stripe rust built based on the spectra with the disease indices greater than 0 acquired in the diseased period of stripe rust in the second group of spectral features. Model 9 was the DI inversion SVR model of wheat leaf rust built based on the spectra with the disease indices greater than 0 acquired in the diseased period of leaf rust in the second group of spectral features. Model 10 was the DI inversion SVR model of wheat stripe rust built based on the spectra with the disease indices greater than 0 acquired in the diseased period of stripe rust in the third group of spectral features. And Model 11 was the DI inversion SVR model of wheat leaf rust built based on the spectra with the disease indices greater than 0 acquired in the diseased period of leaf rust in the third group of spectral features.

rust and leaf rust built based on the spectra with the disease indices greater than 0 acquired in the diseased period in the third group of spectral features were recorded as Model 10 and Model 11, respectively. The optimal parameters $C$ and $\gamma$ for each SVR model and the inversion results were shown in Table 4. As shown in Table 4, the inversion effects of all SVR models were satisfactory. The values of $R^{2}$ and MSE of the testing sets indicated that the inversion effects of the SVR models of wheat leaf rust were better than that of the SVR models of wheat stripe rust. This indicated that wheat canopy hyperspectral data were more suitable for the DI inversion of wheat leaf rust. This may be because the uredinia produced by Prt distribute more evenly on wheat leaves than those produced by Pst.

The results showed that the inversion effects of the SVR models of wheat stripe rust or leaf rust were not greatly improved after preprocessing the spectra data using WPA. Considering the model should be as simple as possible in practical application, it was suggested that monitoring wheat stripe rust and leaf rust can be carried out by using the DI inversion SVR models based on the first group of spectral features.

\section{Conclusions and Discussion}

In this study, the canopy hyperspectral data of healthy wheat, wheat in the incubation period of stripe rust, wheat in the diseased period of stripe rust, wheat in the incubation period of leaf rust, and wheat in the diseased period of leaf rust were obtained. After preprocessing of the data, the disease identification models and the DI inversion models were built based on the three groups of spectral features, respectively. A method based on hyperspectral remote sensing was provided for identification and DI inversion of wheat stripe rust and leaf rust. This study provided some basis for monitoring wheat diseases using satellite remote sensing.

Model 2 based on the second group of spectral features (wavelet packet decomposition coefficients) was better than Model 1 based on the first group of spectral features and Model 3 based on the third group of spectral features. To further improve the identification effect, when one spectrum of wheat canopy is obtained, Model 3 can be firstly used to determine whether the spectrum is obtained from the wheat in the diseased period or not. If yes, Model 4 can be used to identify wheat stripe rust or leaf rust. If not, Model 5 can be used to determine whether wheat is healthy, in the incubation period of stripe rust or in the incubation period of leaf rust. It is difficult to identify healthy wheat, wheat in the incubation period, and wheat in the diseased period of two diseases using only one identification model. An approach using the combination model was provided in this study for solution of this difficulty. The study conducted by Devadas et al. [22] showed that the rust infection caused by $P s t, P r t$, and Pgt in individual wheat leaves could be identified through the combination of two vegetation indices (ARI and TCARI). Therefore, when monitoring and identification of a variety of plant pests are performed by using remote sensing technology, the combination of multiple models can be tried once the purpose cannot be achieved using a single model.

The first group of spectral features used in this study was in a full spectral range $(325-1075 \mathrm{~nm})$. In practice, the specific spectral ranges can be selected for modeling. When the models were built based on the third group of spectral features, all of the 25 feature parameters were used. To reduce the calculation work, the feature parameters with high correlation can be selected for identification and inversion of the diseases.

The results demonstrated that disease indices of wheat stripe rust and leaf rust can be evaluated based on canopy hyperspectral data. The DI inversion SVR models of each wheat disease built in this study could effectively estimate the corresponding disease. In practice, the DI inversion SVR models based on the first group of spectral features should be preferred to estimate wheat stripe rust or wheat leaf rust.

When wheat leaves are infected with Pst or Prt, chlorophyll content in the leaves could be reduced and the changes of cellular structure could be induced; in the later stage of incubation period, chlorotic latent lesions would appear on the leaves; and after incubation period, the uredinia would appear on the leaves and the water content in the leaves would 
be affected $[13,20,38,39]$. These changes caused by disease infection could be detected by spectral features $[5,13]$. In this study, the canopy spectral reflectance of wheat infected with Pst or Prt relatively increased compared with that of healthy wheat in the spectral range $720-1075 \mathrm{~nm}$. This may be induced by the changes of chlorophyll content, cellular structure, and water content. For wheat stripe rust and wheat leaf rust, the shape and distribution of the uredinia on the leaves are different in appearance, and the physiological and biochemical effects on leaves may also be different. So these differences may induce the difference between the canopy spectral reflectance of wheat infected with Pst and that of wheat infected with Prt. Further studies on the relationship between the spectral changes and the physiological and biochemical changes will be helpful for revealing the mechanism of monitoring the two diseases based on hyperspectral data.

With the rapid development of science and technology, hyperspectral remote sensing monitoring technology is increasingly being applied to plant diseases and hyperspectral remote sensing of a variety of plant pests is gradually being concerned. However, many factors, such as leaf angle, soil, background plants, and meteorological conditions, have great influence on the canopy spectral data, and it is very likely to change the relative spectral differences [7]. Therefore, it is very important to find out a method to weaken or eliminate this kind of effects in further studies.

\section{Conflict of Interests}

The authors declare that there is no conflict of interests regarding the publication of this paper.

\section{Authors' Contribution}

Hui Wang and Feng Qin contributed equally to this paper.

\section{Acknowledgments}

This study was supported by National Key Technologies Research and Development Program of China (2012BAD19BA04), National Natural Science Foundation of China (31471726), and International Research Exchange Scheme of the Marie Curie Program of the 7th Framework Program (Ref. PIRSES-GA-2013-612659).

\section{References}

[1] Z. Q. Li and S. M. Zeng, Wheat Rust in China, China Agriculture Press, Beijing, China, 2002.

[2] A. M. Wan, X. M. Chen, and Z. H. He, "Wheat stripe rust in China," Australian Journal of Agricultural Research, vol. 58, no. 6, pp. 605-619, 2007.

[3] W. Chen, C. Wellings, X. Chen, Z. Kang, and T. Liu, "Wheat stripe (yellow) rust caused by Puccinia striiformis f. sp. tritici," Molecular Plant Pathology, vol. 15, no. 5, pp. 433-446, 2014.

[4] R. L. Pu and P. Gong, Hyperspectral Remote Sensing and Its Applications, Higher Education Press, Beijing, China, 2000.

[5] J. S. West, C. Bravo, R. Oberti, D. Lemaire, D. Moshou, and H. A. McCartney, "The potential of optical canopy measurement for targeted control of field crop diseases," Annual Review of Phytopathology, vol. 41, pp. 593-614, 2003.

[6] T. Mewes, J. Franke, and G. Menz, "Spectral requirements on airborne hyperspectral remote sensing data for wheat disease detection," Precision Agriculture, vol. 12, no. 6, pp. 795-812, 2011.

[7] H. G. Wang, J. B. Guo, and Z. H. Ma, "Monitoring wheat stripe rust using remote sensing technologies in China," in Computer and Computing Technologies in Agriculture V, vol. 370 of IFIP Advances in Information and Communication Technology, pp. 163-175, Springer, Berlin, Germany, 2012.

[8] M. Y. Huang, W. J. Huang, L. Y. Liu et al., "Spectral reflectance feature of winter wheat single leaf infected with stripe rust and severity level inversion," Transactions of the CSAE, vol. 20, no. 1, pp. 176-180, 2004.

[9] H. An, H. G. Wang, R. Y. Liu, C. J. Cai, and Z. H. Ma, "Preliminary study on spectral characteristics of single leaf infected by Puccinia striiformis," China Plant Protection, vol. 25, no. 11, pp. 8-11, 2005.

[10] J. L. Zhao, L. S. Huang, W. J. Huang et al., "Hyperspectral measurements of severity of stripe rust on individual wheat leaves," European Journal of Plant Pathology, vol. 139, no. 2, pp. 401-411, 2014.

[11] D. Moshou, C. Bravo, J. West, S. Wahlen, A. McCartney, and H. Ramon, "Automatic detection of 'yellow rust' in wheat using reflectance measurements and neural networks," Computers and Electronics in Agriculture, vol. 44, no. 3, pp. 173-188, 2004.

[12] J. B. Jiang, Y. H. Chen, and W. J. Huang, "Using hyperspectral derivative indices to diagnose severity of winter wheat stripe rust," Optical Technique, vol. 33, no. 4, pp. 620-623, 2007.

[13] J. Zhang, L. yuan, R. Pu, R. W. Loraamm, G. Yang, and J. Wang, "Comparison between wavelet spectral features and conventional spectral features in detecting yellow rust for winter wheat," Computers and Electronics in Agriculture, vol. 100, pp. 79-87, 2014.

[14] L. Y. Liu, M. Y. Huang, W. J. Huang et al., "Monitoring stripe rust disease of winter wheat using multi-temporal hyperspectral airborne data," Journal of Remote Sensing, vol. 8, no. 3, pp. 275281, 2004.

[15] W. J. Huang, D. W. Lamb, Z. Niu, Y. J. Zhang, L. Y. Liu, and J. $\mathrm{H}$. Wang, "Identification of yellow rust in wheat using in-situ spectral reflectance measurements and airborne hyperspectral imaging," Precision Agriculture, vol. 8, no. 4-5, pp. 187-197, 2007.

[16] W. F. Leng, H. G. Wang, Y. Xu, and Z. H. Ma, "Preliminary study on monitoring wheat stripe rust with using UAV," Acta Phytopathologica Sinica, vol. 42, no. 2, pp. 202-205, 2012.

[17] L. Y. Liu, X. Y. Song, C. J. Li, L. Qi, W. J. Huang, and J. H. Wang, "Monitoring and evaluation of the diseases of and yield winter wheat from multi-temporal remotely-sensed data," Transactions of the Chinese Society of Agricultural Engineering, vol. 25, no. 1, pp. 137-143, 2009.

[18] J. B. Guo, C. Huang, H. G. Wang, and Z. H. Ma, "Preliminary study on remote sensing monitoring wheat stripe rust based on SPOT5 image," Acta Phytophylacica Sinica, vol. 36, no. 5, pp. 473-474, 2009.

[19] S. Dutta, S. K. Singh, and M. Khullar, "A case study on forewarning of yellow rust affected areas on wheat crop using satellite data," Journal of the Indian Society of Remote Sensing, vol. 42, no. 2, pp. 335-342, 2014.

[20] D. Ashourloo, M. R. Mobasheri, and A. Huete, "Developing two spectral disease indices for detection of wheat leaf rust (Pucciniatriticina)," Remote Sensing, vol. 6, no. 6, pp. 4723-4740, 2014. 
[21] D. Ashourloo, M. R. Mobasheri, and A. Huete, "Evaluating the effect of different wheat rust disease symptoms on vegetation indices using hyperspectral measurements," Remote Sensing, vol. 6, no. 6, pp. 5107-5123, 2014.

[22] R. Devadas, D. W. Lamb, S. Simpfendorfer, and D. Backhouse, "Evaluating ten spectral vegetation indices for identifying rust infection in individual wheat leaves," Precision Agriculture, vol. 10, no. 6, pp. 459-470, 2009.

[23] L. Yuan, J. C. Zhang, J. L. Zhao, W. J. Huang, and J. H. Wang, "Differentiation of yellow rust and powdery mildew in winter wheat and retrieving of disease severity based on leaf level spectral analysis," Spectroscopy and Spectral Analysis, vol. 33, no. 6, pp. 1608-1614, 2013.

[24] H. B. Qiao, B. Xia, X. M. Ma, D. F. Cheng, and Y. L. Zhou, "Identification of damage by diseases and insect pests in winter wheat," Journal of Triticeae Crops, vol. 30, no. 4, pp. 770-774, 2010.

[25] X. L. Li, Z. H. Ma, L. L. Zhao, J. H. Li, and H. G. Wang, "Early diagnosis of wheat stripe rust and wheat leaf rust using near infrared spectroscopy," Spectroscopy and Spectral Analysis, vol. 33, no. 10, pp. 2661-2665, 2013.

[26] A. A. Gitelson, M. N. Merzlyak, and O. B. Chivkunova, "Optical properties and nondestructive estimation of anthocyanin content in plant leaves," Photochemistry and Photobiology, vol. 74, no. 1, pp. 38-45, 2001.

[27] M. S. Kim, C. S. T. Daughtry, E. W. Chappelle, J. E. McMurtrey, and C. L. Walthall, "The use of high spectral resolution bands for estimating absorbed photosynthetically active radiation (APAR)," in Proceedings of the 6th International Symposium on Physical Measurements and Signatures in Remote Sensing, pp. 299-306, Val-d'Isère, France, 1994.

[28] J. A. Gamon, L. Serrano, and J. S. Surfus, "The photochemical reflectance index: an optical indicator of photosynthetic radiation use efficiency across species, functional types, and nutrient levels," Oecologia, vol. 112, no. 4, pp. 492-501, 1997.

[29] M. N. Merzlyak, A. A. Gitelson, O. B. Chivkunova, and V. Y. Rakitin, "Non-destructive optical detection of pigment changes during leaf senescence and fruit ripening," Physiologia Plantarum, vol. 106, no. 1, pp. 135-141, 1999.

[30] D. Haboudane, J. R. Miller, E. Pattey, P. J. Zarco-Tejada, and I. B. Strachan, "Hyperspectral vegetation indices and novel algorithms for predicting green LAI of crop canopies: modeling and validation in the context of precision agriculture," Remote Sensing of Environment, vol. 90, no. 3, pp. 337-352, 2004.

[31] J. Peñuelas, J. Piñol, R. Ogaya, and I. Filella, "Estimation of plant water concentration by the reflectance Water Index WI (R900/R970)," International Journal of Remote Sensing, vol. 18, no. 13, pp. 2869-2875, 1997.

[32] X. C. Zhang, J. Z. Wu, and Y. Xu, Near-Infrared Spectroscopy and Its Application in Modern Agriculture, Publishing House of Electronics Industry, Beijing, China, 2012.

[33] A. Quinquis, "A few practical applications of wavelet packets," Digital Signal Processing, vol. 8, no. 1, pp. 49-60, 1998.

[34] R. W. Kennard and L. A. Stone, "Computer aided design of experiments," Technometrics, vol. 11, no. 1, pp. 137-148, 1969.

[35] C. Cortes and V. Vapnik, "Support-vector networks," Machine Learning, vol. 20, no. 3, pp. 273-297, 1995.

[36] C. C. Chang and C. J. Lin, "LIBSVM: a library for support vector machines," ACM Transactions on Intelligent Systems and Technology, vol. 2, no. 3, article 27, 2011.
[37] T. M. Cover and P. E. Hart, "Nearest neighbor pattern classification," IEEE Transactions on Information Theory, vol. 13, no. 1, pp. 21-27, 1967.

[38] R. F. Line, "Stripe rust of wheat and barley in North America: a retrospective historical review," Annual Review of Phytopathology, vol. 40, pp. 75-118, 2002.

[39] J. Kuckenberg, I. Tartachnyk, and G. Noga, "Detection and differentiation of nitrogen-deficiency, powdery mildew and leaf rust at wheat leaf and canopy level by laser-induced chlorophyll fluorescence," Biosystems Engineering, vol. 103, no. 2, pp. 121-128, 2009. 

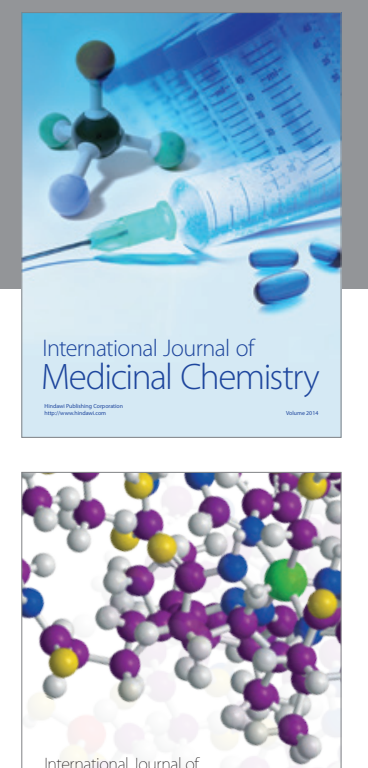

\section{Carbohydrate} Chemistry

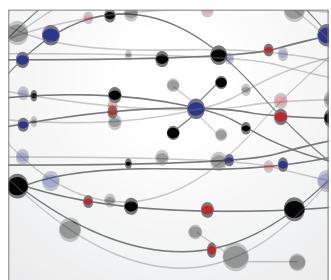

The Scientific World Journal
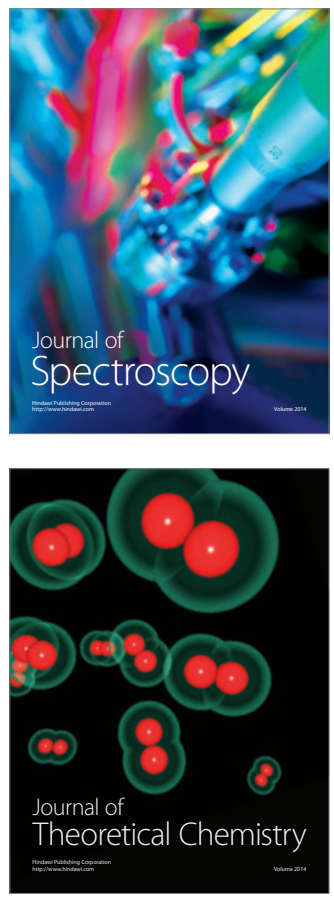
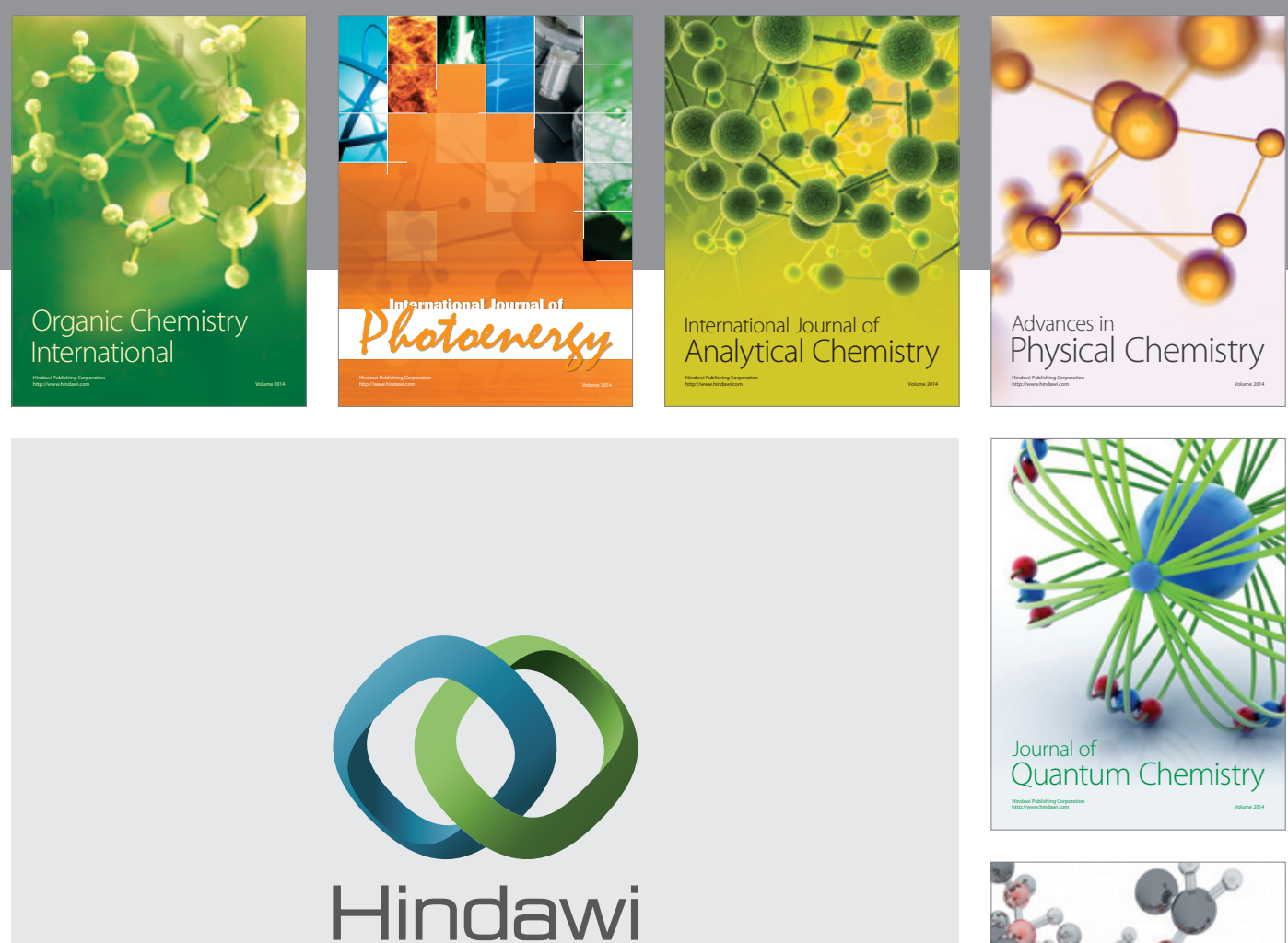

Submit your manuscripts at

http://www.hindawi.com

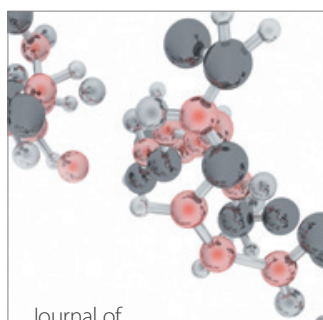

Analytical Methods

in Chemistry

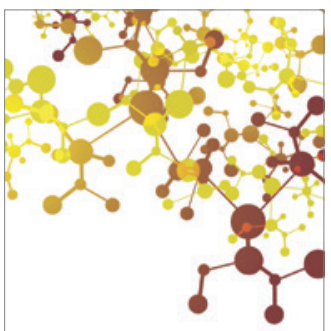

Journal of

Applied Chemistry

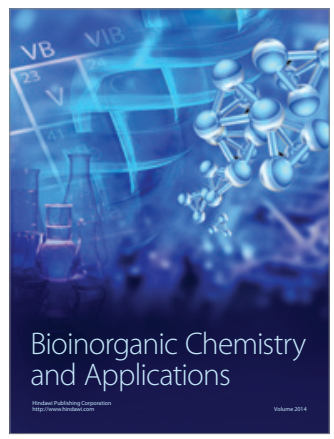

Inorganic Chemistry
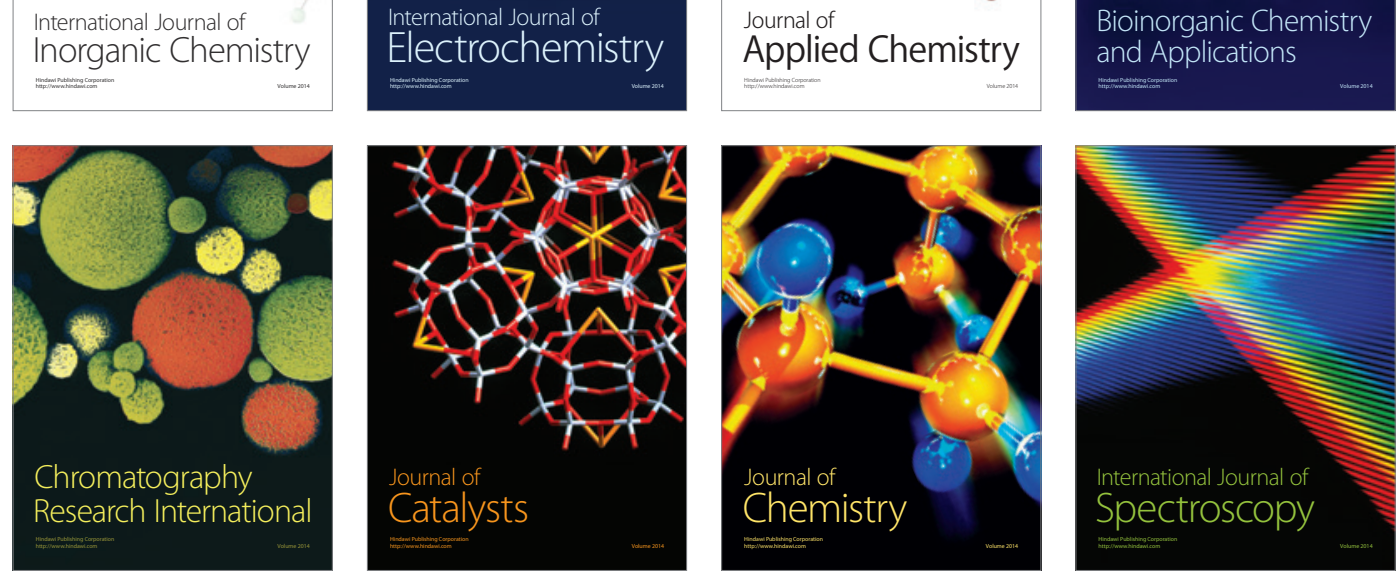